\title{
Le parc urbain des papillons : un dispositif collaboratif de recherche, formation et diffusion des connaissances sur la biodiversité urbaine
}

Magali Deschamps-Cottin, Bruno Vila et Christine Robles

\section{(2) OpenEdition}

Édition électronique

URL : https://journals.openedition.org/ere/4601

DOI : $10.4000 /$ ere.4601

ISSN : 2561-2271

Éditeur

Centr'ERE

Référence électronique

Magali Deschamps-Cottin, Bruno Vila et Christine Robles, « Le parc urbain des papillons : un dispositif collaboratif de recherche, formation et diffusion des connaissances sur la biodiversité urbaine ", Éducation relative à l'environnement [En ligne], Volume 15 - 1 | 2019, mis en ligne le 20 décembre 2019, consulté le 15 octobre 2021. URL : http://journals.openedition.org/ere/4601 ; DOI : https://doi.org/ 10.4000/ere.4601

Ce document a été généré automatiquement le 15 octobre 2021.

La revue Éducation relative à l'environnement est mise à disposition selon les termes de la Licence Creative Commons Attribution - Pas d'Utilisation Commerciale 4.0 International. 


\title{
Le parc urbain des papillons : un dispositif collaboratif de recherche, formation et diffusion des connaissances sur la biodiversité urbaine
}

\author{
Magali Deschamps-Cottin, Bruno Vila et Christine Robles
}

Les auteurs remercient les enseignants du Lycée, les différentes promotions de lycéens et d'étudiants qui ont travaillé au Parc Urbain des Papillons. Ils remercient également les étudiants animateurs qui se sont investis dans ce projet comme les différents partenaires : collectif d'artistes plasticiens intitulé du Sens, de l'Audace, de la Fantaisie et de l'Imagination, Proserpine, Gaz Réseau Distribution France, la Ville de Marseille, Conseil Départemental des Bouches-duRhône, Région Provence-Alpes-Côte d'Azur, Délégations régionales à la recherche et à la technologie Provence-Alpes-Côte d'Azur et le Réseau Culture science Provence-Alpes-Côte d'Azur.

1 La conservation de la biodiversité est devenue un enjeu majeur. Initialement concentrées dans les milieux naturels, les études de la biodiversité se sont progressivement étendues aux zones urbanisées. En effet, au cours des dernières décennies, dans le monde entier, l'urbanisation a entraîné de profonds changements dans le mode d'occupation et d'utilisation des terres (Pickett et coll., 2010). Cette augmentation des zones urbaines a provoqué une artificialisation de nombreux espaces et une fragmentation du paysage qui ont conduit à une profonde altération de la biodiversité. Ce phénomène, déjà important, est toujours en augmentation dans les pays urbanisés (United Nations, 2014). Toutefois, les villes offrent une diversité d'habitats susceptibles d'abriter une diversité d'espèces animales et végétales non négligeable ( McKinney, 2008 ; Robinson et Lundholm, 2012 ; Bonthoux et coll., 2014).

Les travaux réalisés au sein du Laboratoire Population Environnement Développement (LPED, Aix-Marseille Université -IRD) portent depuis 2008 sur la composition, l'organisation, les processus de dispersion et l'homogénéisation de la biodiversité dans 
les espaces urbanisés de Marseille. Ces travaux ont mis en évidence des patrons originaux de répartition d'espèces végétales et animales (Bossu et coll., 2014 ; Lizée, 2011). Les études concernant les communautés de papillons de jour (Lépidoptères Rhopalocères) ont montré une diminution du nombre d'espèces et du nombre d'individus de la périphérie vers le centre-ville. Ainsi, il a été mis en évidence un filtre de sélection des espèces se traduisant par la perte des papillons à affinité méditerranéenne (Lizée et coll., 2011). Il a également été démontré que les pratiques de gestion dans les parcs urbains ou les jardins privatifs viennent accentuer cette sélectivité des espèces au sein des différents contextes urbains de la ville (Lizée et coll., 2014).

3 Sur la base de ces résultats, l'un des auteurs (Magali Deschamps-Cottin) a mis en place en 2012 un dispositif expérimental de recherche, de partage des connaissances et de sensibilisation unique : le Parc Urbain des Papillons (PUP). L'objectif scientifique de ce dispositif était de voir si un aménagement (plantations) et des pratiques de gestions adaptées au cycle de vie des Lépidoptères permettaient le retour et/ou l'arrivée de nouvelles espèces et le maintien des espèces encore présentes sur le site, ou si au contraire, la structure urbaine agissait comme un filtre et ne permettait pas le retour et/ou l'arrivée et donc leur installation durable. (Figure 1). Outre le dispositif expérimental de recherche qu'il constitue, le PUP se veut également un lieu de formation, de médiation et d'échanges à destination de tous les publics : gestionnaires, étudiants, scolaires et grand public. Ainsi, un parcours pédagogique a été pensé et aménagé afin de constituer un support à la médiation scientifique sur l'étude de la biodiversité urbaine.

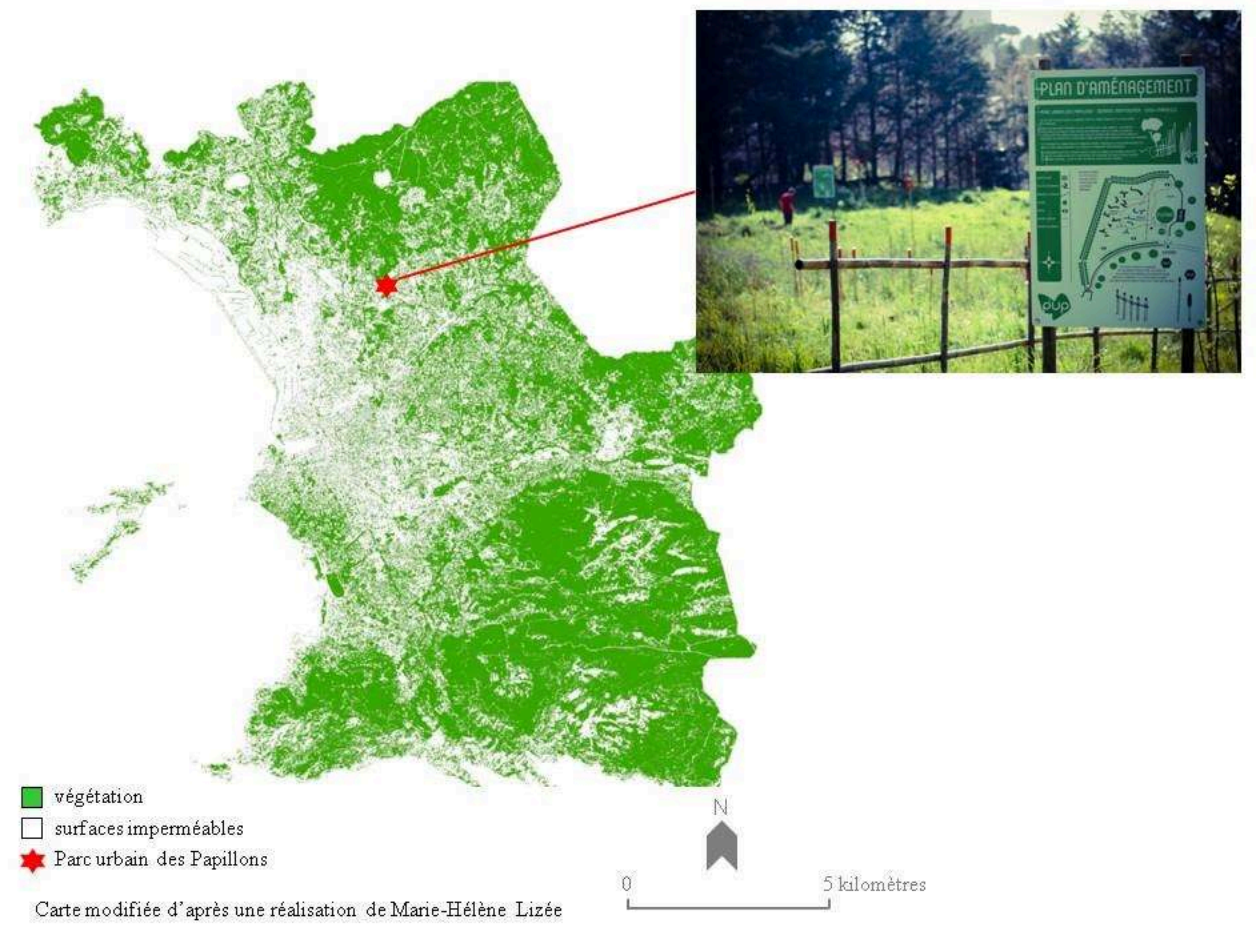

Figure 1 : Localisation du Parc Urbain des Papillons sur la commune de Marseille et photographie du site présentant le plan d'aménagement. (Crédit photo Estel Pierson)

4 L'objectif de cet article est de présenter la construction et la mise en place du programme collaboratif élaboré autour d'une activité de recherche sur la biodiversité 
urbaine et notamment les démarches pédagogiques qui l'accompagnent. En partant du constat scientifique de l'homogénéisation biotique consécutive au processus d'urbanisation (Lizée et coll. 2012, 2014), cet article propose d'exposer 1) comment les scientifiques ont développé leur démarche expérimentale et mis en place le dispositif, 2) comment ils ont fédéré des partenaires variés pour l'aménagement et la coconstruction de leur projet et 3) comment ce dispositif a été pensé et intégré comme un outil dans un parcours de formation et comme un moyen de médiation scientifique avec une dimension pédagogique forte en développant divers supports adaptés aux différents publics. Enfin, il vise à présenter l'aspect réflexif et évolutif en exposant comment la multitude d'acteurs engagée dans ce programme échange et nourri les réflexions autour de cette problématique de la biodiversité urbaine.

\section{De la recherche fondamentale à l'expérimentation : mise en place du projet collaboratif de recherche sur la biodiversité urbaine}

5 Sur la base des résultats de la thèse de Lizée (2011) qui ont montré une différence dans la composition des communautés de papillons en fonction des contextes urbains et des pratiques de gestion dans les parcs urbains, un protocole expérimental a été pensé. Il visait à tester, sur un site donné, l'effet des pratiques de gestion sur le maintien des papillons, voire l'attraction et la colonisation par de nouvelles espèces actuellement absentes des espaces urbanisés marseillais. Pour ce faire, il a été proposé d'une part l'introduction et/ou la réintroduction de certaines plantes hôtes pour les chenilles et de plantes nectarifères pour les adultes et d'autre part, de mettre en place des modes de gestion raisonnés qui visent à conserver les habitats favorables aux papillons à leurs différents stades de cycles de vie.

6 Afin de disposer d'un terrain sur lequel installer durablement le dispositif expérimental, la ville de Marseille a mis à disposition, à la demande des chercheurs, une ancienne friche agricole d'un hectare sur un site qui en comprend 12. Située dans le 14e arrondissement de Marseille, cette friche agricole se trouve dans une zone où le tissu urbain est en cours de densification avec la création de la zone d'activités concertées des Hauts de Sainte-Marthe. Située dans la zone urbanisée périphérique selon les études de Lizée et coll. (2011), cette friche est distante d'un kilomètre du milieu naturel. Plusieurs espèces de papillons présentes dans les milieux naturels adjacents ne s'y observent pas: Pyronia bathseba, Leptotes pirithous, Satyrium esculi, Coenonympha dorus, Lampides boeticus, Charaxes jasius.

7 Un mécène, Gaz Réseau Distribution France, a alors apporté son soutien financier de 2012 à 2015 pour la création du dispositif expérimental (plantations) et les premiers suivis scientifiques (inventaires). À l'aide de son réseau de connaissances professionnelles, l'une d'entre nous a mobilisé différents partenaires pour l'aménagement du PUP. Lors de sa création, l'association Proserpine, pionnière dans la réalisation de Jardins à Papillons (Jardin des Papillons de Digne-les-Bains ${ }^{\circledR}$ ) aidée d'un horticulteur spécialiste dans l'élevage des Lépidoptères a sélectionné et fourni les espèces végétales à planter: plantes hôtes et plantes nectarifères. Le projet s'est renforcé avec le soutien de lycéens issus d'un lycée agricole proposant des formations du certificat professionnel d'aptitude agricole au brevet de technicien supérieur 
agricole autour du paysage, de la nature, de l'environnement et de la maréchalerie. Par la signature d'une convention de Chantier-École pour la formation à la gestion et à l'entretien du parc, le lycée des Calanques, les Lycéens participent à l'entretien et à l'aménagement du site. Un collectif d'artistes plasticiens intitulé du Sens, de l'Audace, de la Fantaisie et de l'Imagination (SAFI) accompagne également le programme dans la mise en œuvre des techniques respectueuses de l'environnement pour l'aménagement du site comme l'utilisation de matériaux naturels (osiers, bambou...) et de techniques de tressage pour la délimitation des parcelles. Ce collectif a également apporté son soutien dans la conception et la réalisation des éléments d'aménagement et l'encadrement des élèves du Lycée dans le cadre d'une Convention de Vie Lycéenne et Apprentie (outil éducatif de la région Provence-Alpes-Côte d'Azur) (Figure 2).

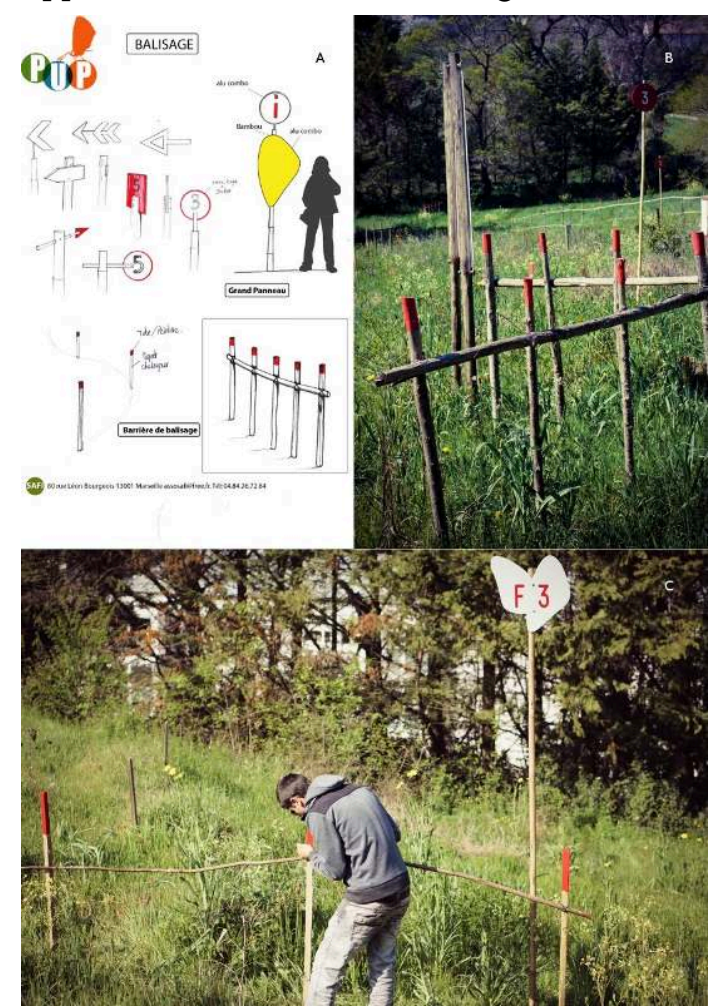

Figure 2 : De l'ébauche à la réalisation, le projet de balisage issu d'une co-construction entre chercheurs, artistes et lycéens. (Crédit photo Estel Pierson, dessin collectif SAFI)

La mobilisation de ces différents acteurs a rapidement donné lieu à de nombreux échanges autour des concepts de biodiversité, des pratiques de gestion, de la place de la nature dans la ville... Ces échanges ont à leur tour donné l'envie aux différents acteurs de partager cette expérience et de diffuser leurs connaissances.

\section{De l'aménagement au parcours pédagogique : mise en place d'un programme d'apprentissage, de formation et de sensibilisation}

Initialement pensé comme un outil de recherche expérimentale sur la biodiversité urbaine, l'aide de lycéens en aménagement paysager a conduit à mettre en place un volet de formation. Ainsi, dès son aménagement, le PUP a intégré un programme 
d'apprentissage et de sensibilisation qui a finalement participé à sa co-construction. À partir d'un parcours pédagogique initial, différentes modalités de fonctionnement ont alors été développées en fonction des publics cibles.

\section{L'aménagement : entre démarche scientifique et pédagogique}

Le Parc Urbain des Papillons a pris place sur une friche agricole présentant plus d'une centaine d'espèces végétales spontanées. Il possède une structure de végétation variée (zones ouvertes, zones arbustives, zones arborées et lisières...) qui offre des habitats diversifiés permettant d'héberger de nombreuses espèces animales.

11 Même si la flore est relativement diversifiée pour une zone urbaine, elle s'avère appauvrie en espèces méditerranéennes du fait de ses usages passés. C'est cette homogénéisation qui explique pour partie la disparition de certaines espèces de papillons à affinité méditerranéenne. Sur la base d'un inventaire floristique, l'aménagement a visé à installer d'autres plantes-hôtes pour les chenilles et d'autres plantes nectarifères pour les adultes afin de compléter la liste des végétaux spontanés. $\mathrm{Au}$ final, 15 massifs ont été plantés ou semés de 53 espèces végétales. De formes variables, ces massifs n'excèdent jamais un mètre de largeur afin de faciliter les observations.

L'aménagement visait aussi à compléter la diversité des habitats en diversifiant les structures présentes sur le site. Tout en conservant pour les papillons des postes de garde, de guet, des solariums et des zones de repos pour les grosses chaleurs, l'aménagement avait pour objectif de préserver des espaces refuges pour tous les stades de développement des papillons (œufs, chenilles, chrysalides et imagos). Ainsi, des zones de friches et des ronciers ont été conservés et des milieux sont maintenus très ouverts par des fauchages annuels.

Dès le début de sa conception scientifique, une démarche pédagogique a été intégrée dans la mise en place du PUP en associant le Lycée des calanques. En formation, les Lycéens ont d'abord trouvé dans ce projet un vaste terrain d'application des pratiques qui leur sont enseignées : désherbage, débroussaillage, plantation, semis, taille... Ce faisant, l'aménagement de plates-bandes diversifiées en terme d'espèces (couleurs des fleurs, familles de plantes, tailles des plantes, disposition des plantes, saison de floraison...) a suscité des questions quant à leur choix. Ceci a nécessité de leur expliquer comment les plantes ont été sélectionnées et de rentrer dans le protocole scientifique. Ils ont alors été formés aux reconnaissances des différentes espèces de Papillons et à leurs besoins vitaux. Cela les a amenés à réfléchir aux conséquences des actions qu'ils réalisaient en matière de gestion. «Si j'arrache cette herbe, est-ce que je n'enlève pas une plante hôte à la chenille de la piéride ? " Formé à une gestion traditionnelle, ils ont tendance à débroussailler et à tout tondre. En reconnaissant les papillons et en apprenant sur leur mode de vie, ils ont rapidement compris que leur gestion supprimait les ronciers qui servaient de poste de guet pour l'Azuré des nerpruns, de plante-hôte pour l'Hespéride de la Mauve et d'abris hivernal pour le Citron de Provence. Ils ont alors pris conscience qu'il fallait conserver des zones avec des ronciers et ne plus tout débroussailler comme ils le faisaient auparavant. Ils ont ainsi requestionné les conséquences de leurs diverses interventions sur le site. Du terrain d'application des pratiques qu'ils étaient venus chercher initialement, les lycéens sont passés à la compréhension de ce qu'ils faisaient et sont finalement devenus acteurs de 
l'aménagement. Ces différents échanges au cours de la mise en place du PUP et le partage des connaissances rendent ces futurs gestionnaires d'espaces verts plus conscients des conséquences de leurs pratiques d'aménagement et de gestion sur la biodiversité, notamment en milieu urbain.

\section{Le parcours pédagogique}

Forts de ces échanges initiaux avec les lycéens, l'idée d'un parcours pédagogique s'est avérée comme une évidence. La création et l'animation du parcours pédagogique a requis les efforts concertés des chercheurs, des membres du collectif SAFI, des lycéens impliqués dans la conception et la gestion du parc et les étudiants en écologie impliqués dans le cadre de la diffusion des connaissances. Le parcours pédagogique a été défini par l'équipe PUP (chercheurs en collaboration avec le collectif SAFI) et est animé par les étudiants sous la supervision des chercheurs.

Le contenu du parcours pédagogique comprend une présentation générale du PUP. Quelles sont les différentes familles de Rhopalocères que l'on peut observer dans le parc ? Quelle est la problématique, quelles sont les connaissances et quelles questions se posent les scientifiques ? Quelle stratégie expérimentale ont-ils imaginée pour y répondre ? Une présentation des aménagements est réalisée. La description de ce qui s'y fait est donnée assez précisément, les suivis des populations de papillons sont expliqués dans les détails. Le parcours pédagogique s'accompagne de démonstrations de capture-recapture, d'identification, de mesures et de suivis.

16 À l'issu du parcours pédagogique qui illustrent différentes connaissances sur les papillons (systématique, cycle de vie, modes de capture...) les élèves et les publics sont invités à participer à la recherche expérimentale menée dans le parc. Pour cela, ils sont invités à échantillonner et à observer, à compter et à chercher le nom des papillons, mais aussi des plantes consommées par ces espèces... Ils peuvent se rendre compte que ce n'est pas facile... De la même façon, pour les étudiants qui suivent une formation au parc, c'est l'occasion d'aborder de manière très concrète de nombreux concepts souvent théoriques qu'ils abordent en cours (cycle de développement, habitats, ressources trophiques...).

\section{Modalités de fonctionnement et supports adaptés aux publics}

17 Pour les scolaires, un programme de découverte de $2 \mathrm{~h}$ à $1 / 2$ journée est proposé sous la forme d'ateliers associant à la présentation du parcours pédagogique des ateliers de manipulation (Figure 3). 


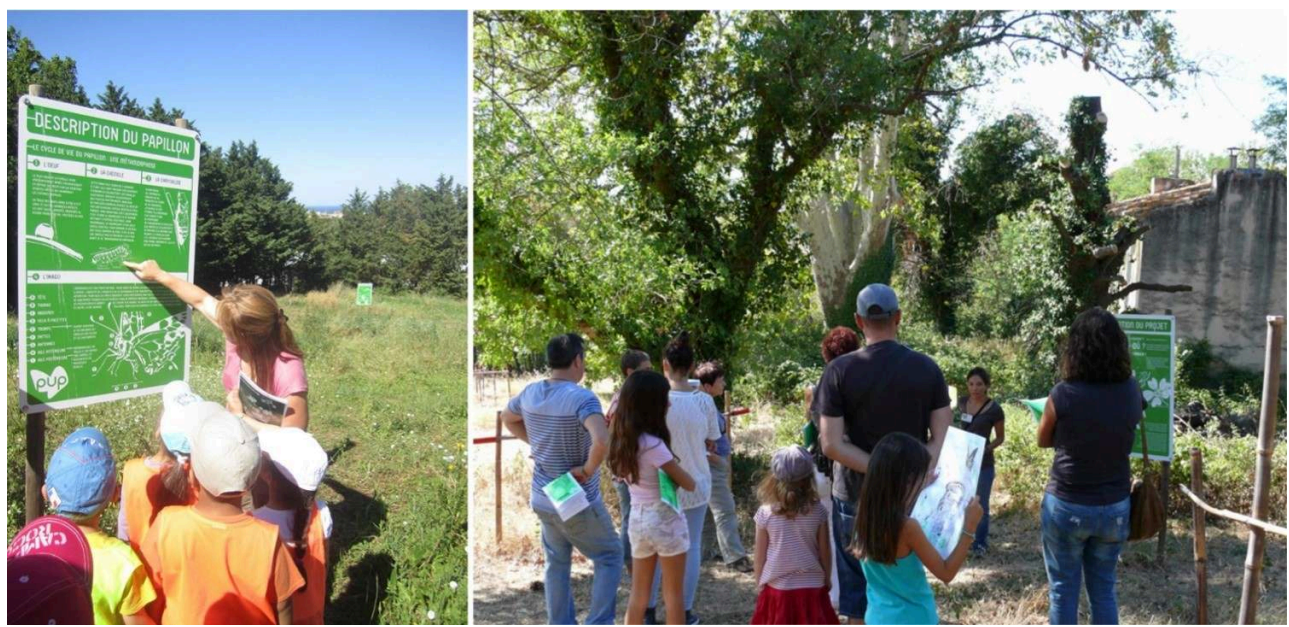

Figure 3 : Animations sur le parcours pédagogique par un enseignant-chercheur et un étudiantmoniteur auprès de scolaires (classe de CP) dans le cadre de la fête de la science 2013 et du grand public lors des journées du Patrimoine de 2013. (Crédit photo Anais Onno)

Pour les scolaires, ce programme de découverte est consolidé par un support papier : « Papillons! Cahier de découverte des lépidoptères sur le Parc Urbain des Papillons ». Ce cahier comporte une partie théorique présentant la morphologie, les grandes familles, le cycle de développement, le régime alimentaire, les habitats.des papillons et une partie pratique et ludique avec des jeux. On peut citer par exemple les activités suivantes :" Retrouve et complète » et «Mots mêlés » qui présentent l'anatomie des papillons ; «Portraits de familles» qui constitue une approche à la biosystématique; «Dessine et relie les points » qui présente un stade de développement; «Fabrique un papillon » correspondant à un origami... (Figure 4). Ce cahier distribué en amont de la visite du parc auprès des professeurs des écoles permet d'introduire différentes notions puis d'en approfondir d'autres à l'issue de la visite.
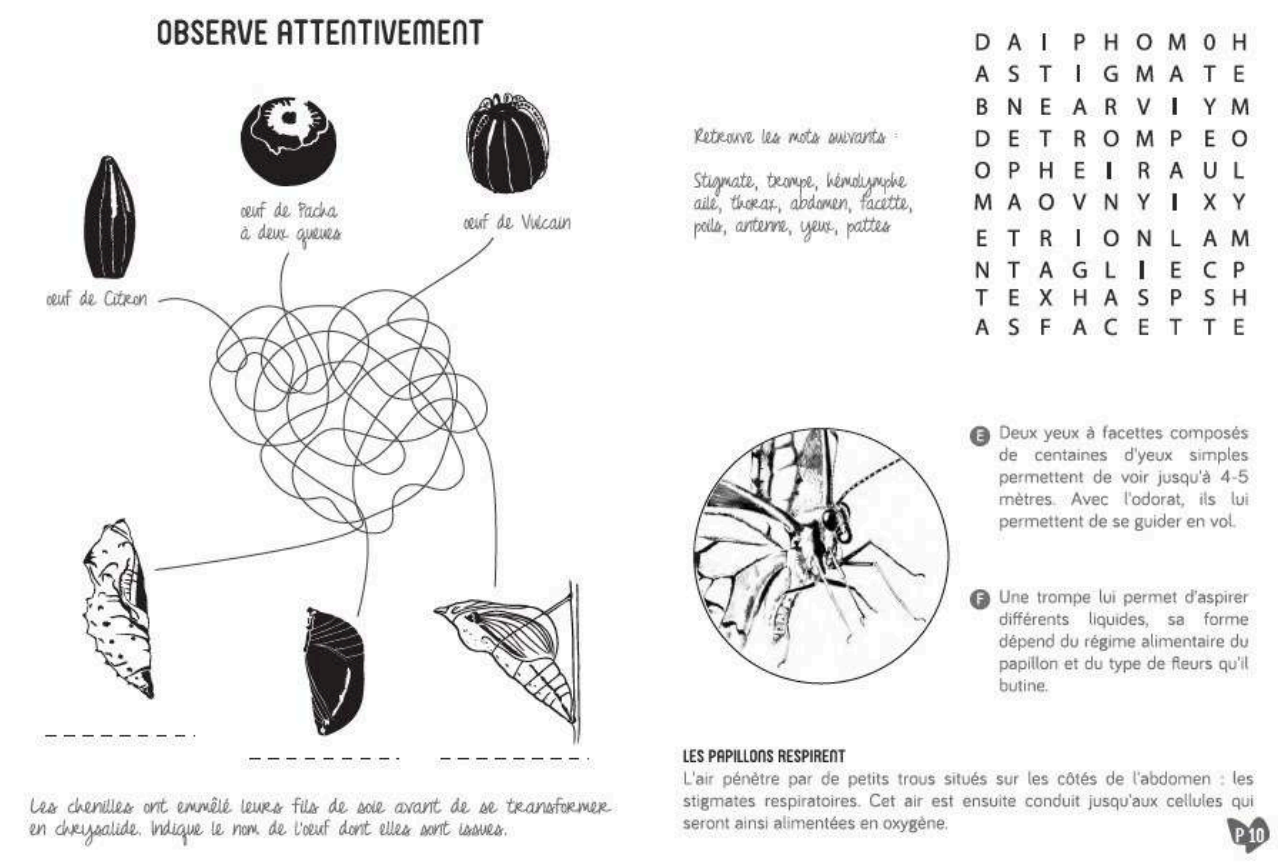

Éducation relative à l'environnement, Volume 15 - 1 | 2019 
Figure 4 : Double page du cahier pédagogique « Papillons! Cahier de découverte des lépidoptères sur le Parc Urbain des Papillons ». à la biodiversité urbaine. Cette sensibilisation prend place lors des manifestations nationales telles que le RDV aux Jardins, la Fête de la science ou les Journées du patrimoine. Elle s'accompagne parfois d'ouvertures en nocturne (par exemple : en 2014, découverte des Papillons de nuit) qui constituent une autre façon de découvrir cette biodiversité et des groupes taxonomiques différents. Parmi le public figurent entre autres des associations de personnes néophytes (Marseille autrement) ou déjà sensibilisées à la nature en ville (Passeur de jardin, La Chevêche, Société d'horticulture et d'arboriculture des Bouches-du-Rhône...). Lors de ces visites, une plaquette est distribuée. Cette plaquette résume, au recto les différentes informations dispensées lors de la visite et au verso, on retrouve un poster contenant les papillons couramment rencontrés au PUP. Ce support permet au visiteur de garder une trace de leur visite tout en étant un premier pas vers la détermination de quelques espèces couramment rencontrées.

Plusieurs promotions d'étudiants inscrits dans le cursus de biologie de la Licence première année au Master (450 étudiants/an) suivent de nombreux enseignements théoriques en systématique, écologie animale et végétale et écologie urbaine. Par l'intégration de la visite de ce parc au sein de leur cursus universitaire, ces étudiants sont initiés à la connaissance naturaliste et à une approche de l'écologie par le terrain et l'expérience. Une forme d'empirisme qui permet également d'aborder la démarche scientifique expérimentale et l'écologie urbaine, mais aussi de mettre en pratique leurs connaissances théoriques sur le terrain.

21 Enfin, des actions de formations concernent les agents des collectivités territoriales (agents techniques des parcs de la ville de Marseille et étudiants du Diplôme d'Etude Supérieur Universitaire «Biodiversité Villes et Territoires»). En visitant le PUP, le parcours pédagogique leur permet d'appréhender des notions dont ils ont entendu parler, mais qu'ils ne connaissent pas forcément (notion de biodiversité, cycles de développement des espèces, habitats...). Le PUP illustre concrètement un dispositif favorisant la biodiversité urbaine par le biais d'aménagements légers et d'une gestion différenciée (Aggeri, 2010). Les résultats des travaux scientifiques menés sur plusieurs années sont exposés. Les agents techniques réfléchissent alors en quoi cette expérimentation est transposable aux modes de gestion des parcs dont ils sont responsables et échangent sur leurs pratiques. Ce qui les amène à questionner des pratiques professionnelles de gestion d'espace de nature en ville et à repenser leurs actions en faveur de la biodiversité.

$\mathrm{Au}$ travers de ce parcours pédagogique, par la mise en place d'un programme d'apprentissage, de formation et de sensibilisation, la démarche expérimentale du PUP prend tout son sens : elle s'enrichit alors des échanges des différents acteurs et donne lieu à de nouvelles plantations et de nouveaux aménagements. Un processus de coconstruction s'engage alors. 


\section{Le Parc Urbain des Papillons, un lieu de réflexion, d'échanges et de partage}

L'ensemble des intervenants et les différents publics sont très motivés par cette approche scientifique expérimentale de terrain et de diffusion scientifique. Lors de la visite du parcours, ils interagissent avec les intervenants (Figure 5). Pour tous, à leurs niveaux respectifs, c'est l'occasion d'aborder et de comprendre différentes notions et concepts théoriques que le parcours permet d'expliquer à l'aide d'exemples concrets. Mais chaque public apporte des éléments propres. C'est surtout le moyen d'ouvrir leur regard sur la présence de Nature même en ville.

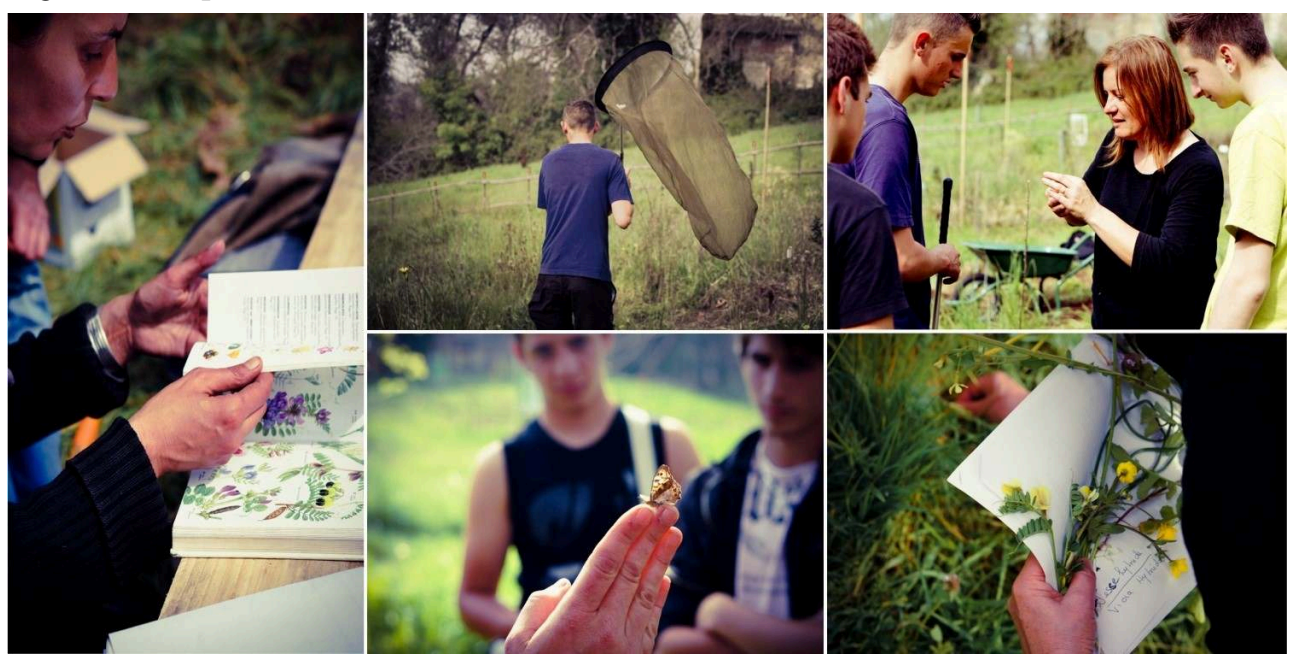

Figure 5 : Illustrations des échanges entre les chercheurs et les différents publics : explications de la démarche scientifique, de l'approche expérimentale ; apprentissage des techniques de suivi (flore, faune) ; mises en pratique et réalisation d'inventaires. (Crédit photo Estel Pierson)

Les scolaires bénéficient d'un service adapté. Des échanges entre les chercheurs, les étudiants animateurs et les professeurs des écoles permettent de définir le contenu des interventions et le déroulement des sessions. Les élèves peuvent acquérir des connaissances particulières en amont de la visite qu'ils reprendront sur le terrain ou bien ces connaissances peuvent être introduites lors de la visite, puis reprises ensuite en classe. Les professeurs qui participent à ces ateliers sont très motivés, car d'une part ils peuvent introduire et illustrer de nombreux concepts de sciences naturelles et pousser la réflexion sur la place de la nature en ville. Ils bénéficient d'un encadrement pour le parcours pédagogique et de support pour préparer ou poursuivre le travail en classe. Certaines idées reçues sont déconstruites, «dingue! un papillon ça ne vie pas qu'une journée ! » ou encore « des papillons migrateurs existent ! « Surprenant! Tout ce qu'on peut apprendre sur les papillons, un grand merci !»

Les collégiens et les lycéens ont la chance de partager leurs idées et de discuter avec les étudiants et les chercheurs. Outre les concepts, ils apprennent à construire une démarche scientifique et voient comment l'expérimentation permet de tester les hypothèses. À l'issue de leur visite, ils sont également invités à réfléchir aux expérimentations réalisées par les chercheurs, à échanger et à apporter leur contribution en proposant des idées sur l'aménagement, une plantation ou des gestes concrets pour le maintien de la biodiversité en ville, ou encore dans leur établissement scolaire « on pourrait construire un PUP dans la cour, il y a plein d'espaces perdus». 
Lors des travaux d'aménagement et d'entretien, les scientifiques amènent par leur protocole expérimental les lycéens professionnels à penser à une gestion différenciée et raisonnée (Figure 6). Une gestion différente appliquée dans certains espaces, en fonction des pratiques des usagers dans ces mêmes espaces, une gestion qui laisse la place aux spontanées. Ils définissent et montrent la biodiversité et, à l'aide d'une approche expérimentale, démontrent comment on l'influence.

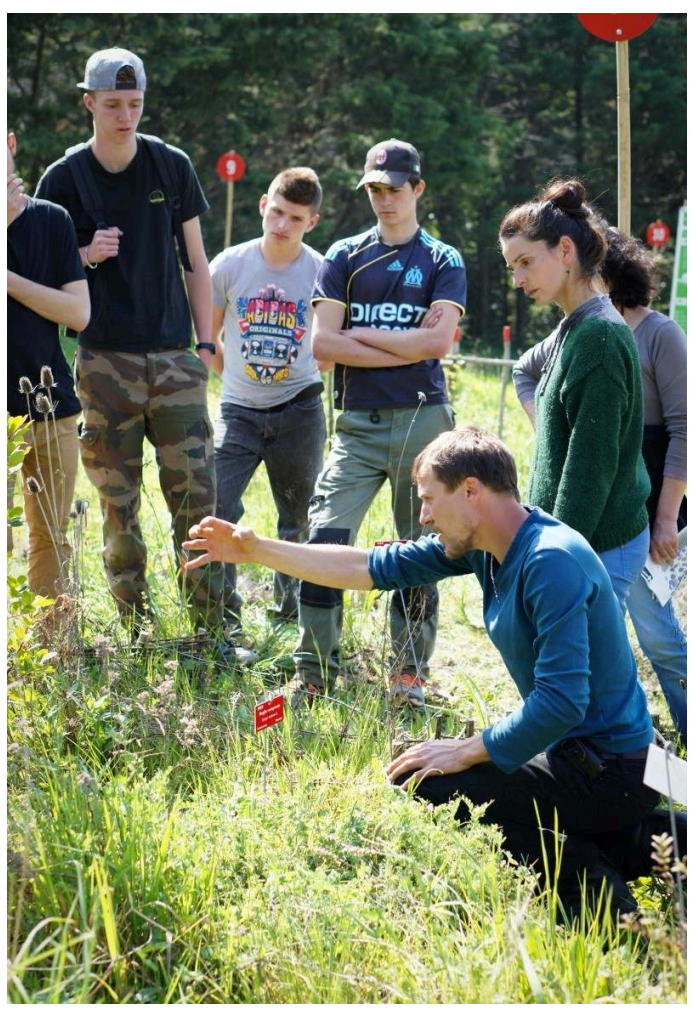

Figure 6 : Professeur du lycée agricole, artiste de l'association SAFI et lycéens échangent autour d'un massif : plantes utilisées, mise en valeur et techniques d'entretien. (Crédit photo Estel Pierson)

Sous la supervision de SAFI, en 2012, leurs réflexions ont donné lieu à différentes propositions de plantations et d'aménagement présentées lors d'une exposition organisée pour la fête de la science à la maison de quartier, puis au lycée (Figure 7). Dans ce cadre, ils ont également réalisé une proposition de logo pour le PUP, un plan pour l'entrée ainsi qu'une signalétique. En 2014, ils ont dessiné et réalisé une zone d'accueil pour les visiteurs. Enfin, en 2016, une autre promotion a proposé et construit un composteur pour le recyclage des déchets végétaux. À chaque fois ces projets résultent des échanges entre les différents acteurs et sont co-construits. 


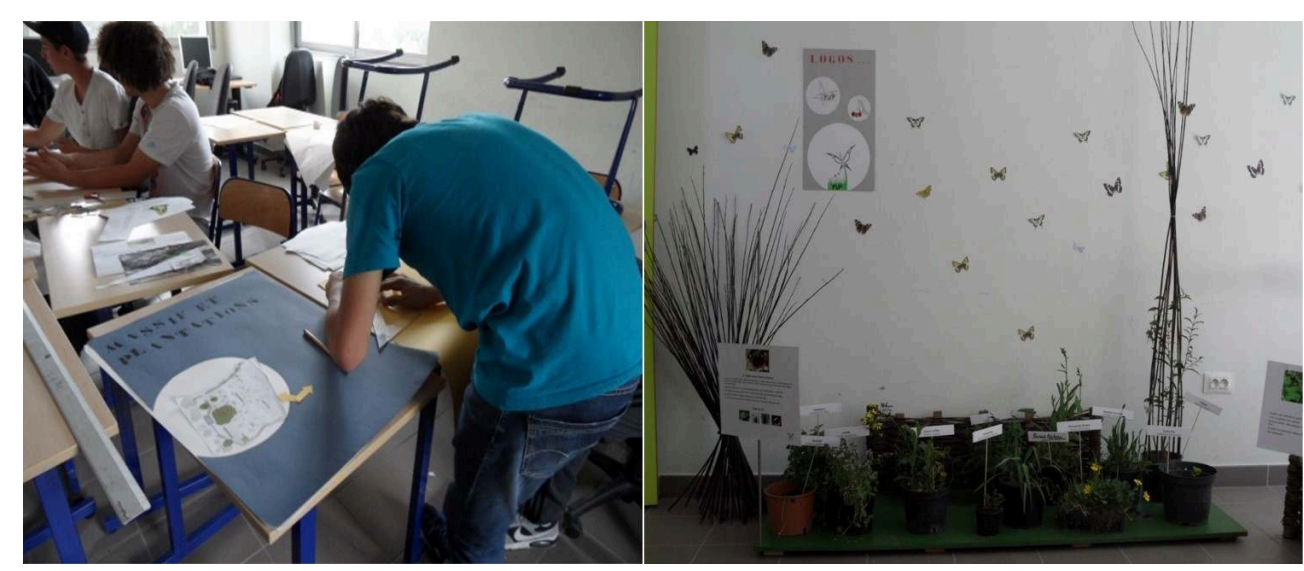

Figure 7 : Préparation d'une exposition par les lycéens présentée lors des journées porte ouverte en 2012 au Lycée agricole. Elle retrace la démarche du projet, ses objectifs ainsi que les échanges des lycéens avec les chercheurs et les artistes dans le cadre de leurs pratiques professionnelles. (Crédit photo Agnès Dechy)

Le grand public est lui très sensible à la conservation de la biodiversité notamment dans les jardins privatifs. Les questions fusent souvent sur la façon de conserver ou de favoriser la diversité dans leur propre jardin. Certains font part de leur expérience personnelle, un dialogue s'instaure à l'issue de chaque parcours comme l'atteste cette citation d'un membre de la Société d'Horticulture et d'Arboriculture des Bouches-duRhône "très instructif! Nous vous aiderons pour les plantations de nectarifères". Ainsi, le parcours pédagogique n'est pas figé et évolue au cours des années en fonction des remarques et questions des visiteurs. Un processus de co-construction s'amorce alors. En réponse à la question "pourquoi suivre les papillons », un panneau sur son rôle dans la chaine alimentaire a été rajouté. Un panneau supplémentaire est venu compléter les premières informations, en particulier sur la façon de mettre en œuvre à l'échelle des jardins privatifs des plantations en faveur des papillons et des insectes en général (Figure 8). Cette initiative a été reprise au sein du livret pédagogique sous l'intitulé «Invite les papillons ». Inversement, des visiteurs ont proposé des plantes qu'ils cultivent comme plantes hôtes pour des plantations sur le PUP : « vous n'avez pas de fenouil pour le Machaon, nous en avons plein dans notre jardin, nous vous en apporterons » ou encore proposent leur aide pour l'entretien « Merci pour votre temps passé pour conserver ces zones protégées. Les personnes du quartier apprécient ces activités. Nous restons à votre disposition pour vous aider à entretenir la zone ». 


\section{ATTIRER LES PAPILLONS}

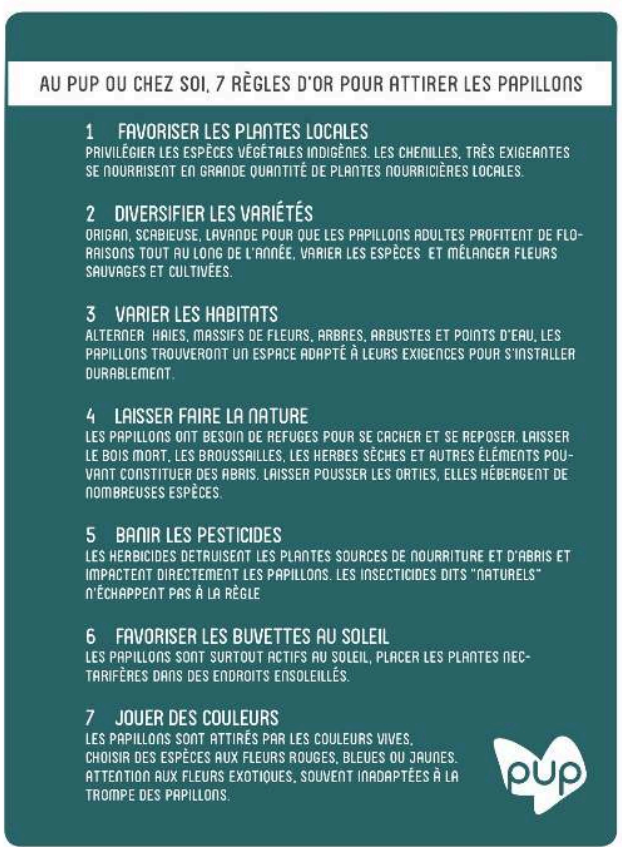

Figure 8 : Panneau rajouté dans le parcours pédagogique, résultant des échanges du public avec les chercheurs et relatif aux conseils à mettre en œuvre pour l'attraction des papillons dans les jardins privatifs notamment.

Avec ce parcours pédagogique, les agents techniques des parcs de la ville de Marseille et ceux suivant la formation du DESU appréhendent de manière concrète les effets des recommandations qu'on leur demande de mettre en œuvre. Ce parcours leur permet de voir comment de petits actes permettent un maintien ou une perte de biodiversité. Ils échangent leurs propres expériences et mesurent l'influence de leur intervention sur la biodiversité. Cela les amène en retour à réfléchir sur leurs interventions et à les repenser. Ces échanges devraient déboucher sur la rédaction d'une charte préservation de la biodiversité ordinaire en ville.

Enfin, les étudiants les plus intéressés et motivés réalisent des stages au sein du PUP. Stages qu'ils réalisent sur des sujets divers en fonction de leur projet professionnel. Leur formation scientifique s'affine au cours des années universitaires. En retour, en fonction de leurs compétences naturalistes, ils complètent les inventaires taxonomiques du site. À titre d'exemple des inventaires d'insectes pollinisateurs et d'escargots ont été réalisés respectivement en 2015 et 2016. Les étudiants qui animent le parcours pédagogique sont formés avant leur première séance et sont rémunérés pour les animations réalisées. Ils acquièrent une expérience pédagogique précieuse pour leurs curriculums vitae et une meilleure compréhension du processus d'enseignement et de diffusion des connaissances scientifiques... Cette expérience est différente de leur expérience antérieure en tant que vacataires ou comme attaché temporaire d'enseignement où ils donnent des cours théoriques avec de faibles interactions avec les élèves. 


\section{Conclusion}

31 Avec le Parc Urbain des Papillons, le public, les élèves et les étudiants ont l'occasion de s'engager dans un apprentissage actif. L'intérêt d'un tel programme est l'aspect collaboratif et la sensibilisation à la biodiversité urbaine à travers un modèle sensible, esthétique et attrayant que peut représenter le Papillon. À partir d'un enjeu de société que représente la conservation de la biodiversité en ville auquel les scientifiques tentent de répondre, ce programme s'est considérablement enrichi des expériences et des échanges des différents acteurs. En passant de spectateurs à acteurs, ces différents publics sont davantage sensibilisés et sentent qu'ils peuvent agir à leur niveau. Les gestionnaires, agents techniques appréhendent concrètement leur action dans le maintien de la biodiversité en ville tandis que les scolaires, collégiens et lycéens appréhendent une démarche scientifique expérimentale. Les scientifiques quant à eux se confrontent à d'autres expérimentations conduites par des visiteurs et accèdent à d'autres formes d'innovations parfois empiriques et explorent des pistes qu'ils n'auraient pas forcément abordées ou traitées sous cet angle.

Au final, ce sont environ 1600 étudiants de première année sur 3 ans, une vingtaine d'agents techniques de la ville de Marseille ainsi qu'une cinquantaine d'autres collectivités, 140 élèves du primaire, 164 collégiens et lycéens et pas moins de 430 personnes grand public qui ont suivi le parcours pédagogique du PUP et échangés. Après plus de 6 ans d'existence, le PUP est en activité. La recherche scientifique et la sensibilisation des publics se poursuivent avec toujours comme ambition une approche collaborative et une démarche engagée et militante. Comme le souligne Audrey Muratet et François Chiron dans le Manuel d'écologie urbaine (2019), il est primordial de reconnecter les citadins avec la nature et «La construction socio-écologique des espaces de nature en ville permet d'enrayer leur disparition et favorise un engagement citoyen en faveur de leur conservation ».

\section{BIBLIOGRAPHIE}

Aggéri, G. (2010) Inventer les villes-natures de demain... Gestion différenciée, gestion durable des espaces verts. Dijon : Educagri éditions

Bonthoux, S., Brun, M., Di Pietro, F., Greulich, S. et Bouché-Pillon, S. (2014) How can wastelands promote biodiversity in cities? A review. Landscape and urban planning 132, 79-88.

Bossu, A., Marco, A., Manel, S. et Bertaudière-Montes, V. (2014) Effects of built landscape on taxonomic homogenization: Two case studies of private gardens in the French Mediterranean. Landscape and urban planning 129, 12-21.

Lizée, M. H., Bourdil, C., Barthélémy et Deschamps-Cottin, M. (2014) Approche socio-écologique des parcs publics marseillais. Méditerranée 123, 123-132. 
Lizée, M.H., Manel, S., Mauffrey, J.F., Tatoni, T. et Deschamps-Cottin. M. (2012) Matrix configuration and patch isolation influences override the species-area relationship for urban butterfly communities. Landscape Ecology 27, 159-169.

Lizée, M.H. (2011) Diversité, organisation spatiale et fonctionnelle des communautés de Papillons (Lépidoptères, Rhopalocères) en milieu urbain et périurbain. Rôle des espaces artificialisés en termes de conservation et de connectivité. Thèse de l'Université Aix-Marseille I, Marseille, le 8 décembre, $249 p+$ annexes.

Lizée, M.H., Mauffrey, J.F, Tatoni, T. et Deschamps-Cottin, M. (2011) Monitoring urban environments on the basis of biological traits, Ecological indicators 11, 353-361.

McKinney, M.L. (2008) Effects of urbanization on species richness: A review of plants and animals. Urban Ecosystems 11, 161-176.

Muratet, A., Chiron, F., Muratet, M. (2019) Manuel d'écologie urbaine. Dijon : Éditions « Les presses du réel ».

Pickett, S.T.A., Cadenasso, M.L., Grove, J.M., Boone, C.G., Groffman, P.M., Irwin, E., Kaushal, S.S., Marshall, V., McGrath, B.P. et Nilon, C.H. (2010) Urban ecological systems : Scientific foundations and a decade of progress. Journal of Environmental Management 92, 331-361.

Robinson, S.L. et Lundholm, J.T. (2012) Ecosystem services provided by urban spontaneous vegetation. Urban Ecosystems 15, 545-557.

UN (2014) World urbanization prospects - The 2014 revision. Published by the United Nations, Department of Economic and Social Affairs, Population Division. ISBN 978 - 92-1-151517

\section{RÉSUMÉS}

Dans les zones urbaines du monde entier, les scientifiques ont observé l'homogénéisation de la biodiversité. De plus, l'urbanisation et les modes de vie modernes ont considérablement réduit les connaissances et les interactions de l'homme avec la nature. Ces deux phénomènes ont de graves conséquences pour la conservation de la biodiversité. Dans cet article, nous présentons la conception et la mise en œuvre, par un large éventail d'acteurs, d'un dispositif expérimental de recherche et de sensibilisation sur la biodiversité urbaine, le Parc Urbain des Papillons (PUP). L'élaboration collaborative de ce dispositif associe, d'une part, la recherche expérimentale pour repenser les pratiques de gestion des parcs urbains afin de favoriser le maintien voire la reconquête de la biodiversité dans les espaces urbains et, d'autre part, la formation participative, la médiation et les échanges avec un public diversifié (gestionnaires de l'environnement, étudiants, scolaires et grand public). L'intérêt de ce dispositif réside dans l'aspect collaboratif, permettant un enrichissement par échanges d'expériences entre les différents acteurs et les publics. Pour conclure, le PUP offre de nombreuses opportunités de partager et d'échanger différemment dans le domaine des sciences du vivant et de développer des programmes d'apprentissage, de formation et de sensibilisation largement négligés en milieu urbain.

Throughout the world, in urban areas, scientists have observed the homogenization of the biodiversity. Furthermore, urbanization and modern lifestyles have greatly reduced people's knowledge of and interaction with nature. Both have grave consequences for the conservation of the biodiversity. Here, we present the construction and the implementation, by a wide range of actors, of a park focused on urban biodiversity, the Parc Urbain des Papillons (PUP). The collaborative construction of this park associates, on one hand, experimental research on rethinking management practices to encourage the reestablishment of biodiversity in the city 
and, on the other hand, active training, mediation and exchanges for a range of publics (environment managers, students, schoolchildren and the general public). The interest of this kind of programme lies in the collaborative aspect, allowing considerable enrichment through the exchange of experiences between the various actors and publics. We conclude by pointing out that this experimental park provides extensive opportunities for sharing science differently and for developing learning, training and outreach programmes overlooked in urban areas.

\section{INDEX}

Keywords : Learning, sharing science, biodiversity, experimental park, urban area

Mots-clés : apprendre, partager les connaissances, biodiversité, dispositif expérimental, espaces urbains

Index géographique : France

\section{AUTEURS}

\section{MAGALI DESCHAMPS-COTTIN}

Écologue, maître de conférences à Aix-Marseille Université au LPED, Magali Deschamps-Cottin s'intéresse aux dynamiques des communautés animales dans les écosystèmes anthropisés par l'étude des mécanismes de leur maintien en relation avec les modes de gestion et le degré de naturalité des espaces. Ses recherches sont majoritairement conduites en interdisciplinarité (avec sociologues, géographes, urbanistes) en collaboration avec des gestionnaires, ce qui l'a conduit à créer un site de recherche et de formation pour tous publics : le Parc Urbain des Papillons. (Courriel : magali.deschamps-cottin[@]univ-amu.fr)

\section{BRUNO VILA}

Écologue, maître de conférences à Aix-Marseille Université au LPED, Bruno Vila s'intéresse aux dynamiques des communautés végétales, à la plasticité des espèces ainsi qu'à la conservation en particulier dans les écosystèmes anthropisés. Il s'intéresse aux assemblages d'espèces afin d'élaborer un indicateur de naturalité urbaine. Ses recherches abordent également un aspect diachronique de la flore urbaine avec l'utilisation des herbiers comme outils originaux pour aborder les dynamiques urbaines. (Courriel : bruno.vila[@]univ-amu.fr)

\section{CHRISTINE ROBLES}

Écologue, maître de conférences à Aix-Marseille Université au LPED, Christine Robles développe certains outils de l'écologie fonctionnelle susceptibles de fournir des éléments d'explication à la dynamique de la biodiversité en milieu urbain et aux notions d'homogénéisation biotique et de connectivité paysagère. Ses recherches s'articulent autour de la stratégie d'allocation des ressources et de la phénologie des espèces avec également la prise en compte du compartiment sol en tant que variable explicative supplémentaire des communautés végétales urbaines. (Courriel : christine.robles[@]univ-amu.fr) 